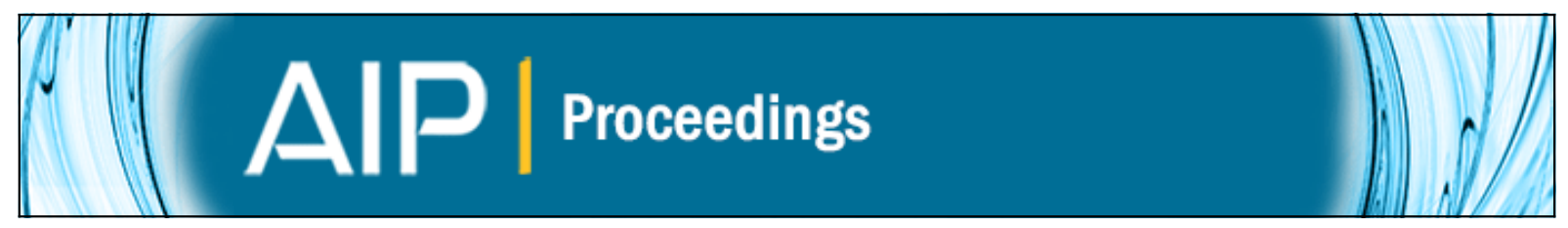

What does cosmology tell us about particle physics beyond the Standard Model?

Eiichiro Komatsu

Citation: AIP Conference Proceedings 1467, 151 (2012); doi: 10.1063/1.4742093

View online: http://dx.doi.org/10.1063/1.4742093

View Table of Contents: http://scitation.aip.org/content/aip/proceeding/aipcp/1467?ver=pdfcov

Published by the AIP Publishing

Articles you may be interested in

Cosmological constraints on the roll parameter of freezing and thawing quintessence models

AIP Conf. Proc. 1548, 254 (2013); 10.1063/1.4817054

Beyond the standard model with precision nucleon matrix elements on the lattice

AIP Conf. Proc. 1441, 540 (2012); 10.1063/1.3700610

Sneutrino inflation in supersymmetric B - $L$ with inverse seesaw

AIP Conf. Proc. 1467, 294 (2012); 10.1063/1.4742121

Natural GUT and the cosmology

AIP Conf. Proc. 1467, 135 (2012); 10.1063/1.4742090

Searching for particle physics beyond the standard model at the LHC and elsewhere

AIP Conf. Proc. 1446, 9 (2012); 10.1063/1.4727987 


\title{
What does cosmology tell us about particle physics beyond the Standard Model?
}

\author{
Eiichiro Komatsu \\ Texas Cosmology Center and the Department of Astronomy, The University of Texas at Austin, 1 University \\ Station, C1400, Austin, TX 78712, USA \\ Kavli Institute for the Physics and Mathematics of the Universe, Todai Institutes for Advanced Study, the \\ University of Tokyo, Kashiwa, Japan 277-8583 (Kavli IPMU, WPI) \\ Max-Planck-Institut für Astrophysik, Karl-Schwarzschild-Str. 1, 85741 Garching, Germany
}

\begin{abstract}
Cosmology demands particle physics beyond the Standard Model: we need to explain the nature of dark matter and dark energy, and the physics of cosmic inflation. Cosmology also provides the tightest upper bound on the sum of neutrino masses, and it seems only a matter of time before we measure the absolute mass of neutrinos, unveiling the neutrino mass hierarchy. It also provides a measurement of the number of relativistic species at the photon decoupling epoch (at which the temperature of the universe is $3000 \mathrm{~K}$ ). Astronomy and Astrophysics Decadal Survey conducted by USA's National Academy of Sciences has identified these four topics (dark matter, dark energy, inflation and neutrinos) as the most important subjects to study in cosmology over the next decade. In this contribution, we review the current status on these topics, in light of the recent cosmological constraints.
\end{abstract}

Keywords: cosmology; cosmic microwave background; dark matter; dark energy; inflation; neutrino mass PACS: $98.80 .-\mathrm{k}$

\section{INTRODUCTION}

Do we need particle physics beyond the Standard Model? From cosmologist's point of view, the answer to this question is a solid "yes," as we need to explain the nature of dark matter and dark energy, as well as the physics of cosmic inflation. Explaining any of these requires new physics beyond the Standard Model.

Combining temperature and polarization anisotropies of the cosmic microwave background measured by the Wilkinson Microwave Anisotropy Probe (WMAP) satellite [1] and the other astrophysical data that are sensitive to the cosmic distance scales (such as the Baryon Acoustic Oscillation (BAO) [2] and the Hubble constant $\left(H_{0}\right)$ [3]), we have determined the energy budget of the current universe to be $4.58 \pm 0.16 \%$ hydrogen and helium nuclei; $22.9 \pm 1.5 \%$ dark matter; and $72.5 \pm 1.6 \%$ dark energy [4]. While we know that dark matter and dark energy exist, we do not know what they are. There are no particles in Standard Model which can account for the measured mass density of dark matter. (The mass density of the standard left-handed neutrinos is at most $10 \%$ of the measured mass density of dark matter.) The leading candidate for dark energy is a cosmological constant (or vacuum energy), but it tells us nothing as to why the observed vacuum energy is so small compared to a native expectation from quantum field theory.

The current data of the cosmic microwave background are consistent with the standard predictions of inflation. The spatial curvature of the observable universe is zero: the dimensionless curvature parameter is constrained to be $-0.013<\Omega_{k}<0.008$ (positive $\Omega_{k}$ being negative curvature) at $95 \% \mathrm{CL}$. The primordial fluctuations are nearly scaleinvariant and Gaussian. The precise definition of these terms (scale invariance and Gaussianity) will be provided in "Inflation" section below, but the parameters that characterize these properties are constrained as $n_{s}=0.96 \pm 0.01$ $(68 \% \mathrm{CL})[4,5,6]$ and $f_{\mathrm{NL}}=32 \pm 21(68 \% \mathrm{CL})$ [4]. Some inflation models have been ruled out by the data, but the others have survived. We do not yet know what physics drove inflation.

From a host of neutrino oscillation experiments, we now know that at least two neutrino mass eigenstates have non-zero masses (see references in [7]). As these experiments measure only the mass squared differences between the neutrino mass eigenstates $i$ and $j$, i.e., $m_{i}^{2}-m_{j}^{2}$, we do not yet know the absolute mass of neutrinos. Cosmology has been playing a leading role in constraining the sum of neutrino masses, $\sum_{i} m_{i}$. In particular, as we argue in "Mass of Neutrinos" section below, determining whether $\sum_{i} m_{i}=0.06 \mathrm{eV}$ or $\sum_{i} m_{i}=0.1 \mathrm{eV}$ has an important implication for the neutrino mass hierarchy. The current most robust $95 \%$ CL upper bound on $\sum_{i} m_{i}$ from cosmology (the WMAP 7-year data combined with $\mathrm{BAO}$ and $\left.H_{0}\right)$ is $\sum_{i} m_{i}<0.58 \mathrm{eV}$ [4]. It seems only a matter of time before we actually measure 

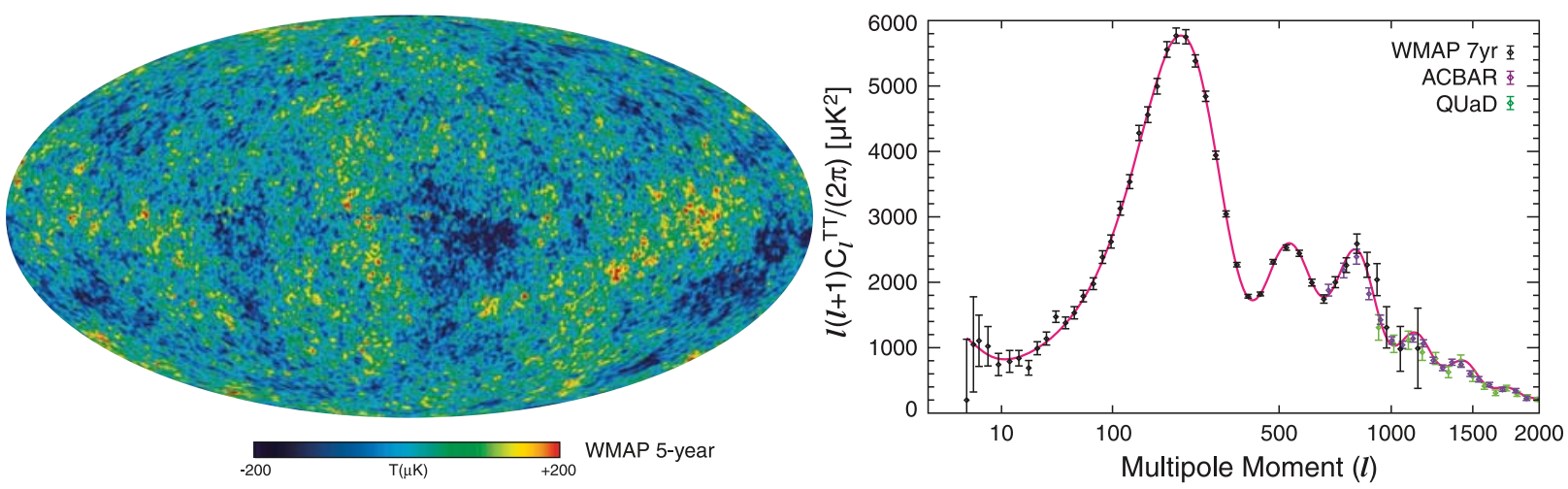

FIGURE 1. Temperature anisotropy of the cosmic microwave background measured by WMAP. (Left) Full-sky temperature map (smoothed to angular resolution of 1 degree full-width-at-half-maximum) of the WMAP 5-year data in Galactic coordinates after subtracting Galactic foreground emission using 5 frequency bands [10]. (Right) Angular power spectrum of the WMAP 7-year temperature data (black points with error bars), $C_{l}^{T T}=(2 l+1)^{-1} \sum_{m}\left|a_{l m}\right|^{2}$, where $a_{l m}$ is the spherical harmonics coefficients of the temperature map, $T(\hat{n})$, computed from $a_{l m}=\int d \hat{n} T(\hat{n}) Y_{l m}(\hat{n})$ [4]. The purple and green points with error bars are ground-based data from the ACBAR [11] and the QUaD [12] experiments, respectively.

$\sum_{i} m_{i}$ at the level of $0.06 \mathrm{eV}[8]$.

LEP's precision measurements of the decay width of $Z$ bosons into neutrinos (which is measured from the total decay width minus the decay width to quarks and charged leptons) shows that there are only 3 left-handed neutrino species [9]. In cosmology, these neutrinos act as massless particles (i.e., relativistic particles) before the temperature of the universe falls below $T \simeq m_{i} / 2.25$. On the other hand, there may be other neutrino species (such as right-handed neutrinos) which are inactive or invisible at colliders. There may also be other relativistic particles in the universe which are yet to be found. One can combine the observed abundance of helium nuclei with theory of the Big Bang Nucleosynthesis to determine the number of relativistic species at the temperature of $10^{9} \mathrm{~K}$. One can use the observed cosmic microwave background anisotropy to determine the number of relativistic species at the temperature of $3000 \mathrm{~K}$. There is a tentative $2-\sigma$ hint that there may be one extra relativistic degree of freedom (in addition to photons and 3 neutrino species) at $3000 \mathrm{~K}[4,5,6]$. It is too early to draw any definitive conclusions about this; however, if confirmed by, e.g., the Planck data which are sensitive enough to detect such an extra relativistic species at 5- $\sigma$ level, then it would have a profound implication for particle physics beyond the Standard Model.

The latest "Astronomy and Astrophysics Decadal Survey" conducted by USA's National Academy of Sciences (known as Astro2010) has identified these four topics (dark matter, dark energy, inflation and neutrinos) as the most important subjects to study in cosmology over the next decade. ${ }^{1}$ In this contribution, we shall review the current status on these topics, in light of the recent cosmological constraints (mainly from the WMAP 7-year data shown in Figure 1). Unless noted otherwise, the parameter values quoted below are from [4].

\section{DARK MATTER DENSITY}

In order to fit the power spectrum of temperature anisotropy, $C_{l}^{T T}$, shown in the right panel of Figure 1, we need two matter components: one is the baryonic matter (and electrons) which interact with photons, creating the acoustic oscillation vividly seen in the measured $C_{l}^{T T}$. The other is the dark matter which interacts with the other components or with itself only gravitationally, and is "cold" in a sense that it is pressureless with zero sound speed. (On the other hand, the photon-baryon fluid has a sound speed of $c_{s}=\frac{c}{\sqrt{3(1+R)}}$ where $R \equiv \frac{3 \rho_{b}}{4 \rho_{\gamma}}$.) As the inferred mass density of dark matter is 5 times as large as that of baryons, the basic picture here is that photons and baryons interact with each other forming the acoustic oscillation in a gravitational potential well provided mainly by dark matter.

Roughly speaking, we determine the baryon density to the photon density ratio, $\frac{\rho_{b}}{\rho_{\gamma}}$, from the ratio of the heights of

\footnotetext{
${ }^{1}$ See page T-3 of Astro2010 Panel Report for recommendations made by "Cosmology and Fundamental Physics Committee," which was chaired by David N. Spergel at Princeton University.
} 
the first and second acoustic peaks, and the total matter density (i.e., baryon plus dark matter) to the total radiation density (i.e., photons plus neutrinos) ratio, $\frac{\rho_{b}+\rho_{d m}}{\rho_{\gamma}+\rho_{v}}$, from the ratio of the heights of the first and third acoustic peaks. As the photon energy density is given (in natural units) by $\rho_{\gamma}=\frac{\pi^{2}}{15} T_{\gamma}^{4}$ and we know the photon density precisely from the measurement of the present-day photon temperature $(2.725 \pm 0.001 \mathrm{~K}$ [13]), the first-to-second peak ratio gives the baryon density parameter as $\Omega_{b}=0.0458 \pm 0.0016(68 \% \mathrm{CL})$. The neutrino energy density is given by $\rho_{v}=\frac{7}{8} \frac{\pi^{2}}{15} N_{\mathrm{eff}} T_{v}^{4}$ (where a factor of $7 / 8$ is due to Pauli's exclusion principle). Assuming the standard thermal history of the universe, the neutrino temperature, $T_{V}$, is related to the photon temperature, $T_{\gamma}$, as $T_{V}=(4 / 11)^{1 / 3} T_{\gamma}$, and the effective number of neutrino species is given by $N_{\text {eff }}=3.04$. With these assumptions, the first-to-third peak ratio gives the dark matter density parameter as $\Omega_{d m}=0.229 \pm 0.015(68 \%$ CL).

However, once we relax the assumption of $N_{\text {eff }}=3.04$ and consider a possibility of adding extra relativistic species (which do not have to have anything to do with neutrinos), then we can no longer constrain $\Omega_{d m}$ from the WMAP data alone. On the other hand, if we have information on $\Omega_{d m}$ from other observations (such as the large-scale structure of

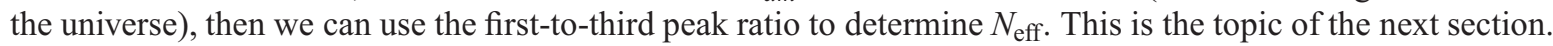

\section{NUMBER OF RELATIVISTIC SPECIES}

When we talk about the "extra relativistic degrees of freedom," it is customary to treat these extra degrees of freedom as if they were neutrinos. In other words, we parametrize the extra relativistic energy density, $\rho_{R}$, as

$$
\rho_{v}+\rho_{R} \rightarrow \tilde{\rho}_{v}=\frac{7}{8} \frac{\pi^{2}}{15} N_{\mathrm{eff}} T_{v}^{4}
$$

with $N_{\text {eff }} \geq 3.04$ and $T_{v}=(4 / 11)^{1 / 3} T_{\gamma}$. As a result, $N_{\text {eff }}$ does not have to be an integer: if these extra species are Bosons, the number would be different by a factor of $7 / 8$. Their temperature can certainly be different from $(4 / 11)^{1 / 3} T_{\gamma}$.

How can we constrain the effective number of relativistic degrees of freedom, $N_{\text {eff }}$ ? We can use the following properties to constrain $N_{\text {eff: }}$ :

1. Energy density. Adding extra relativistic species increases the radiation energy density. This in turn delays the matter-radiation equality (the epoch at which the total matter density and the total radiation density are equal), increasing the height of the first acoustic peak. It also increases the expansion rate of the universe at the time of photon decoupling via the Friedmann equation:

$$
H^{2}=\frac{8 \pi G}{3}\left(\rho_{m}+\rho_{\gamma}+\tilde{\rho}_{v}\right)
$$

This effect enhances damping of power at $l>1000$ seen in the right panel of Figure $1[14,15]$ (i.e., the power at $l>1000$ will damp more for a larger value of $N_{\text {eff }}$ ).

2. Anisotropic stress. Relativistic particles which do not interact directly with other particles or themselves cannot be described as a (perfect) fluid. As a result, the distribution function, $f(\mathbf{x}, \mathbf{p}, t)$, of relativistic particles have a non-negligible anisotropic stress, $\pi_{i j}$, given by

$$
\pi_{i j} \equiv \int \frac{d^{3} p}{(2 \pi)^{3}} p\left(\hat{p}_{i} \hat{p}_{j}-\frac{1}{3} g_{i j}\right) f(\mathbf{x}, \mathbf{p}, t),
$$

as well as higher-order moments. This then changes the metric perturbation via Einstein's field equations. As a result, the power spectrum of the cosmic microwave background is reduced almost equally at $l>200[14,16]$.

In Figure 2, we show how $N_{\text {eff }}=7$ changes the temperature power spectrum.

In 2009, we have used the WMAP 5-year data to detect the effect of the neutrino anisotropic stress for the first time [17]. In 2011, we have combined the WMAP 7-year data with the BAO and $H_{0}$ data to measure $N_{\text {eff }}=4.3 \pm 0.9$ $(68 \%$ CL) [4], which uses the matter-radiation equality epoch constraint and the anisotropic stress effects. However, WMAP's angular resolution is not quite sufficient to detect a change in the damping tail of the power spectrum.

Recently, South Pole Telescope (SPT) and Atacama Cosmology Telescope (ACT), ground-based experiments which are more sensitive to the power spectrum on smaller angular scales, have used all of the above three effects to tighten the limit on $N_{\text {eff }}$ to $N_{\text {eff }}=4.6 \pm 0.8\left(68 \%\right.$ CL; ACT) [6] and $N_{\text {eff }}=3.85 \pm 0.62(68 \%$ CL; SPT) [5]. 


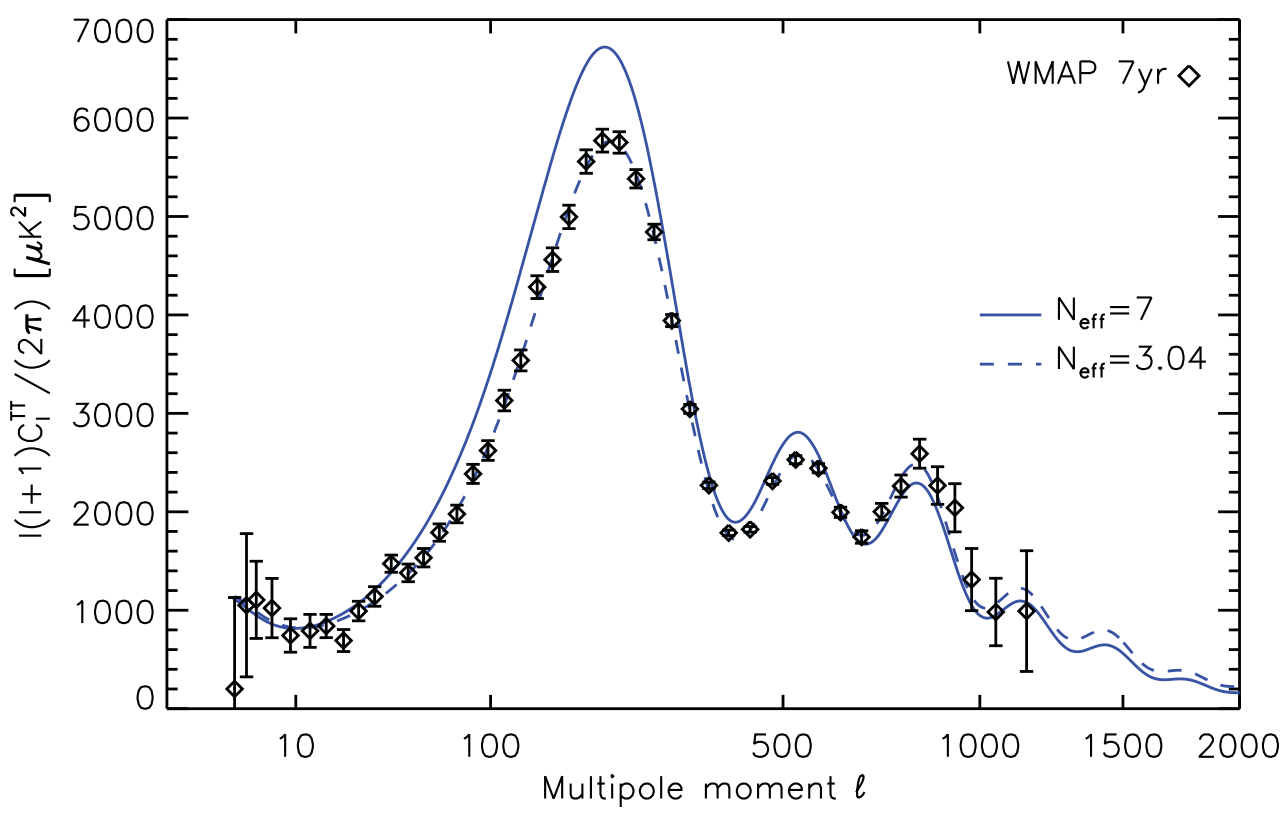

FIGURE 2. Effect of $N_{\text {eff }}=7$ on the temperature power spectrum of the cosmic microwave background (solid line). The dashed line shows the standard case with $N_{\text {eff }}=3.04$. The first peak is enhanced due to a delayed matter-radiation equality epoch, whereas the power at higher multipoles is suppressed more due to a larger anisotropic stress and a larger expansion rate of the universe at the photon decoupling epoch. Note that the horizontal axis of the solid line has been multiplied by a factor of 0.895 so that both lines have the same position of the third peak.

The forthcoming Planck data are expected to reach the $68 \% \mathrm{CL}$ error bar of $\Delta N_{\text {eff }}=0.2$; thus, it should be able to detect $N_{\text {eff }}=4$ at the 5- $\sigma$ level. It would then have profound implications for particle physics beyond the Standard Model.

\section{MASS OF NEUTRINOS}

How about the mass of neutrinos? Among three neutrino mass eigenstates, the mass squared difference between the mass eigenstates 1 and 2 has been determined as $m_{2}^{2}-m_{1}^{2}=(7.62 \pm 0.19) \times 10^{-5} \mathrm{eV}^{2}(68 \% \mathrm{CL})$ [18]; thus, $m_{1}<m_{2}$. On the other hand, only the absolute value of the mass squared difference between the mass eigenstates 1 and 3 has been measured, and thus we do not yet know whether $m_{3}$ is greater or smaller than $m_{1}$. As $\left|m_{3}^{2}-m_{1}^{2}\right| \gg m_{2}^{2}-m_{1}^{2}$, we have two possibilities:

1. Normal hierarchy: $m_{1}<m_{2}<m_{3}$

2. Inverted hierarchy: $m_{3}<m_{1}<m_{2}$

For the normal hierarchy, the mass squared difference between the mass eigenstates 1 and 3 has been determined as $m_{3}^{2}-m_{1}^{2}=\left(2.53_{-0.10}^{+0.08}\right) \times 10^{-3} \mathrm{eV}^{2}(68 \% \mathrm{CL})$, whereas for the inverted hierarchy, $m_{3}^{2}-m_{1}^{2}=-\left(2.40_{-0.07}^{+0.10}\right) \times 10^{-3} \mathrm{eV}^{2}$ $(68 \%$ CL) [18]. Therefore, for the minimal scenario in which the lightest mass eigenstate is massless, the sum of neutrino masses is given by $\sum_{i} m_{i}=0.06 \mathrm{eV}$ for the normal hierarchy and $\sum_{i} m_{i}=0.1 \mathrm{eV}$ for the inverted hierarchy. This level of neutrino mass is well within reach of cosmological experiments (especially the large-scale structure) [8]. If the minimum neutrino mass is greater than $0.1 \mathrm{eV}$, then the mass hierarchy is degenerate, i.e., $m_{1} \simeq m_{2} \simeq m_{3}$.

How well can the cosmic microwave background constrain the mass of neutrinos? We do not expect massive neutrinos to affect the the cosmic microwave background power spectrum very much (except through the gravitational lensing effect), if they were still relativistic at the photon decoupling epoch. This means that, for massive neutrinos to affect the the cosmic microwave background power spectrum, at least one of the neutrino masses must be greater 


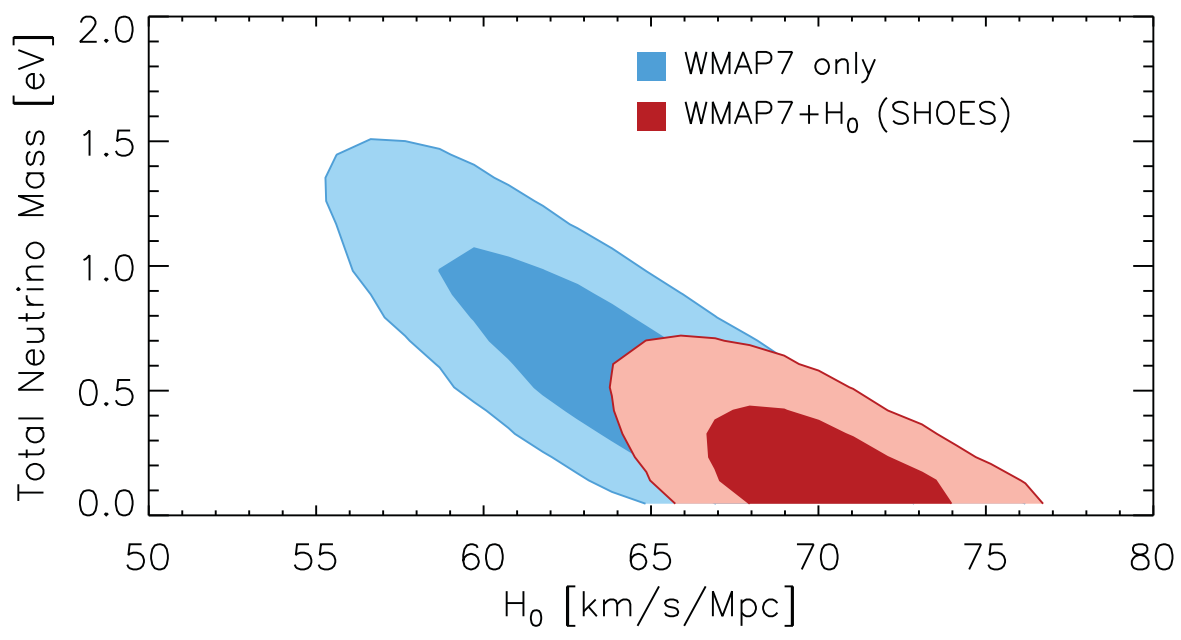

FIGURE 3. Constraint on the total mass of neutrinos, $\sum_{i} m_{i}$. The larger contours are for the WMAP 7-year-only constraint, while the smaller contours are for the WMAP 7-year combined with the constraint on $H_{0}=74.2 \pm 3.6 \mathrm{~km} / \mathrm{s} / \mathrm{Mpc}$ (68\% CL) from the SHOES collaboration [3]. The inner and outer contours show the $68 \% \mathrm{CL}$ and $95 \% \mathrm{CL}$ regions, respectively.

than the mean energy of relativistic neutrinos per particle when the photon temperature of the universe was $T_{\gamma} \simeq$ $3000 \mathrm{~K} \simeq 0.26 \mathrm{eV}$. Since the mean energy of relativistic neutrinos is given by $\langle E\rangle=\left(7 \pi^{4} T_{v}\right) /(180 \zeta(3)) \simeq 3.15 T_{V}=$ $3.15(4 / 11)^{1 / 3} T_{\gamma}$, we need at least one neutrino species whose mass satisfies $m_{v}>3.15(4 / 11)^{1 / 3} T_{\gamma} \simeq 0.58 \mathrm{eV}$; thus, it would not be possible to constrain the neutrino mass using the the cosmic microwave background data alone, if the mass of the heaviest neutrino species is below this value.

If the neutrino mass eigenstates are degenerate with the effective number of species equal to 3.04, this argument suggests that $\sum m_{v} \sim 1.8 \mathrm{eV}$ would be the limit to which the the cosmic microwave background data are sensitive. Ichikawa et al. [19] argue that $\sum m_{v} \sim 1.5 \mathrm{eV}$ would be the limit for the the cosmic microwave background data alone, which is fairly close to the value given above.

In order to go beyond $\sim 1.5 \mathrm{eV}$, therefore, one needs to combine the the cosmic microwave background data with the other cosmological probes. The constraint on $H_{0}$ is especially useful for improving the limit compared to the WMAPonly limit (see Figure 3). The WMAP 7-year data combined with the BAO and $H_{0}$ have yielded $\sum_{i} m_{i}<0.58 \mathrm{eV}$ [4].

What information does $H_{0}$ add to improve the limit on $\sum_{i} m_{i}$ ? This effect has been explained by Ichikawa et al. [19] as follows.

The massive neutrinos modify the power spectrum by their changing the matter-to-radiation ratio at the decoupling epoch. If the sum of degenerate neutrino masses is below $1.8 \mathrm{eV}$, the neutrinos were still relativistic at the decoupling epoch. However, they are definitely non-relativistic at the present epoch, as the neutrino oscillation experiments have shown that at least one neutrino species is heavier than $0.05 \mathrm{eV}$. This means that the $\Omega_{m}$ that we measure must be the sum of $\Omega_{b}, \Omega_{d m}$, and $\Omega_{v}$; however, at the decoupling epoch, neutrinos were still relativistic, and thus the matter density at the decoupling epoch was actually smaller than a naive extrapolation from the present value.

As the matter-to-radiation ratio was smaller than one would naively expect, it would accelerate the decay of gravitational potential around the decoupling epoch. This leads to an enhancement in the so-called early integrated Sachs-Wolfe (ISW) effect. The larger $\sum_{i} m_{i}$ is, the larger early ISW becomes, as long as the neutrinos were still relativistic at the decoupling epoch, i.e., $\sum_{i} m_{i}<1.8 \mathrm{eV}$.

The large ISW causes the first peak position to shift to lower multipoles by adding power at $l \sim 200$; however, this shift can be absorbed by a reduction in the value of $H_{0} \cdot{ }^{2}$ This is why $\sum_{i} m_{i}$ and $H_{0}$ are anti-correlated.

\footnotetext{
${ }^{2}$ This is similar to what happens to the curvature constraint from the WMAP data alone. A positive curvature model, $\Omega_{k}<0$, shifts the acoustic peaks to lower multipoles; however, this shift can be absorbed by a reduction in the value of $H_{0}$. As a result, a closed universe with $\Omega_{k} \sim-0.3$ and
} 


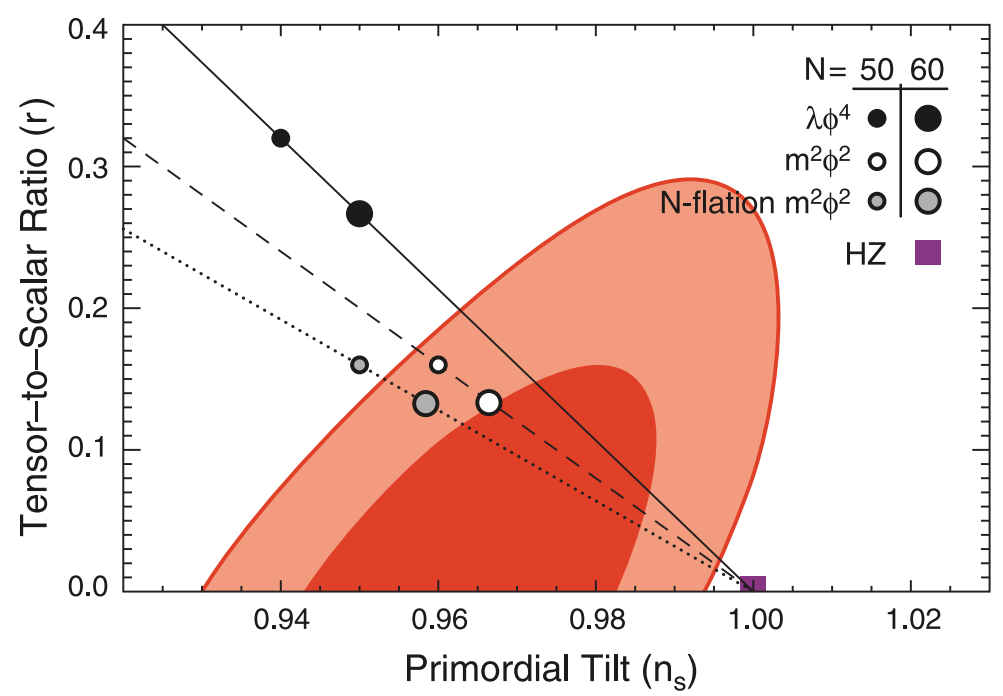

FIGURE 4. Two-dimensional joint marginalized constraint ( $68 \%$ and $95 \% \mathrm{CL}$ ) on the spectral index of the primordial power spectrum, $n_{s}$, and the tensor-to-scalar ratio, $r$, derived from the data combination of $W M A P+\mathrm{BAO}+H_{0}$. The symbols show the predictions from "chaotic" inflation models whose potential is given by $V(\phi) \propto \phi^{\alpha}$ [21], with $\alpha=4$ (solid) and $\alpha=2$ (dashed) for single-field models, and $\alpha=2$ for multi-axion field models with $\beta=1 / 2$ (dotted; [22]). This figure is adopted from [4].

\section{PHYSICS OF INFLATION}

Inflation is thought to have occurred at the energy scale of Grand Unification $\left(\approx 10^{16} \mathrm{GeV}\right)$, and thus any constraints on inflation from cosmology are directly testing our understanding of physics at such high energies that the particle colliders would never reach. There are three basic observables which are useful for distinguishing between various inflation models:

Scale-invariant power spectrum Quantum fluctuations during inflation produce perturbations to the metric. Let a perturbation to the trace-part of the space-space metric be $\mathscr{R}$. One may write the space-space metric as $g_{i j}=a^{2}(t) e^{2 \mathscr{R}}\left[e^{h}\right]_{i j}$ with $\operatorname{det}\left[e^{h}\right]_{i j}=1$ (hence $\operatorname{Tr}[h]=0$ ). Let us then define the power spectrum of $\mathscr{R}$ as $\left\langle\mathscr{R}_{\mathbf{k}} \mathscr{R}_{\mathbf{k}^{\prime}}\right\rangle=(2 \pi)^{3} \delta_{D}\left(\mathbf{k}+\mathbf{k}^{\prime}\right) P_{\mathscr{R}}(k) .\left(\delta_{D}(x)\right.$ is the Dirac delta function.) The "scale-invariant power spectrum" is defined by

$$
P_{\mathscr{R}}(k) \propto k^{-3} .
$$

In reality many inflation models predict a nearly, but not exactly, scale-invariant spectrum. We usually parametrize the power spectrum as

$$
P_{\mathscr{R}}(k) \propto k^{n_{s}-4}
$$

with $n_{s} \approx 1$. The current data suggest $n_{s}=0.96 \pm 0.01(68 \% \mathrm{CL})[4,5,6]$.

Tensor-to-scalar ratio The presence of primordial gravitational waves, $h_{i j}$ (which is the transverse and traceless part of the space-space metric from $\left[e^{h}\right]_{i j}=1+h_{i j}+\mathscr{O}\left(h^{2}\right)$ ), is a robust prediction of inflation models, as the same mechanism that generated $\mathscr{R}$ should also generate primordial gravitational waves [23,24]. The amplitude of gravitational waves relative to that of $\mathscr{R}$ is model-dependent. Let us define the power spectrum of the gravitational wave, $h_{i j}$, as $\left\langle h_{i j, \mathbf{k}} h^{i j, \mathbf{k}^{\prime}}\right\rangle=(2 \pi)^{3} \delta_{D}\left(\mathbf{k}+\mathbf{k}^{\prime}\right) P_{h}(k)$. It is then customary to parametrize the amplitude of gravitational waves using the so-called "tensor-to-scalar ratio," $r$, defined by

$$
r \equiv \frac{P_{h}\left(k_{0}\right)}{P_{\mathscr{R}}\left(k_{0}\right)},
$$

where $k_{0}=0.002 \mathrm{Mpc}^{-1}$ is some reference wavenumber at which $r$ is defined. We have not detected primordial gravitational waves from inflation yet, and the current upper bound is $r<0.24$ (95\% CL) [4]. In particular,

$\Omega_{\Lambda} \sim 0$ is still a good fit, if Hubble's constant is as low as $H_{0} \sim 30 \mathrm{~km} / \mathrm{s} / \mathrm{Mpc}[20]$. 
the $n_{s}-r$ space is useful for constraining various inflation models, as different models make different, testable predictions for a combination of these parameters. In Figure 4, we show the current constraint on the $n_{s}-r$ plane along with some representative inflation models. For example, a single-field inflation model with a scalar-field potential of $V(\phi) \propto \phi^{4}$ has been ruled out by the current data. This is remarkable - this shows that inflation has now become a testable science.

Gaussianity Inflation generates $\mathscr{R}$ as a quantum fluctuation. Single-field inflation models usually predict that the probability distribution of $\mathscr{R}$ is nearly a Gaussian distribution. To be more precise, the three-point function (called the bispectrum):

$$
\left\langle\mathscr{R}_{\mathbf{k}_{1}} \mathscr{R}_{\mathbf{k}_{2}} \mathscr{R}_{\mathbf{k}_{3}}\right\rangle=(2 \pi)^{3} \delta_{D}\left(\mathbf{k}_{1}+\mathbf{k}_{2}+\mathbf{k}_{3}\right) B_{\mathscr{R}}\left(k_{1}, k_{2}, k_{3}\right),
$$

vanishes for Gaussian fluctuations. (I.e., $B_{\mathscr{R}}\left(k_{1}, k_{2}, k_{3}\right)=0$ for a Gaussian $\mathscr{R}$.) Therefore, any non-zero threepoint function is a signature of non-Gaussianity. Single-field inflation models usually predict

$$
\frac{6}{5} f_{\mathrm{NL}} \equiv \frac{B_{\mathscr{R}}\left(k_{1}, k_{2}, k_{3}\right)}{P_{\mathscr{R}}\left(k_{1}\right) P_{\mathscr{R}}\left(k_{2}\right)+(2 \text { perm. })} \ll 1 .
$$

In general $f_{\mathrm{NL}}$ defined in this way may depend on wavenumbers. The $f_{\mathrm{NL}}$ that does not depend on wavenumbers is often called the "local form." It has been shown that the local-form $f_{\mathrm{NL}}$ is related to $n_{s}$ via $f_{\mathrm{NL}}=\frac{5}{12}\left(1-n_{s}\right) \approx 0.02$ for all single-field models regardless of the details of models [25, 26, 27, 28, 29, 30, 31]. Therefore, a detection of $f_{\mathrm{NL}} \gg 1$ would rule out all single-field inflation models (except for some subtlety regarding the initial vacuum state of quantum fluctuations: see $[32,33,34])$. As we have not detected the local-form $f_{\mathrm{NL}}$ yet, the current data are consistent with the prediction of single-field inflation models. The current bound on $f_{\mathrm{NL}}$ is $f_{\mathrm{NL}}=32 \pm 21$ $(68 \%$ CL) [4]. The Planck data are expected to reduce the error bar by a factor of four, further testing single-field inflation models [35].

\section{ACKNOWLEDGMENTS}

We would like to thank the WMAP team for a wonderful collaboration over so many years. The WMAP mission is made possible by the support of the Science Mission Directorate Office at NASA Headquarters. This work is additionally supported in part by NASA grant NNX08AL43G and NSF grants AST-0807649 and PHY-0758153.

\section{REFERENCES}

1. C. Bennett, et al., Astrophys.J. 583, 1-23 (2003), astro-ph/0301158.

2. W. J. Percival, et al., Mon.Not.Roy.Astron.Soc. 401, 2148-2168 (2010), 0907.1660.

3. A. G. Riess, et al., Astrophys.J. 699, 539-563 (2009), 0905.0695.

4. E. Komatsu, et al., Astrophys.J.Suppl. 192, 18 (2011), 1001.4538.

5. R. Keisler, et al., Astrophys.J. 743, 28 (2011), 1105.3182.

6. J. Dunkley, et al., Astrophys.J. 739, 52 (2011), 1009.0866.

7. M. Maltoni, T. Schwetz, M. Tortola, and J. Valle, New J.Phys. 6, 122 (2004), hep-ph/ 0405172.

8. M. Takada, E. Komatsu, and T. Futamase, Phys.Rev. D73, 083520 (2006), astro-ph/ 0512374.

9. K. Nakamura, et al., J.Phys. G G37, 075021 (2010).

10. G. Hinshaw, et al., Astrophys.J.Suppl. 180, 225-245 (2009), 0803.0732.

11. C. Reichardt, et al., Astrophys.J. 694, 1200-1219 (2009), 0801.1491.

12. M. Brown, et al., Astrophys.J. 705, 978-999 (2009), 0906.1003.

13. D. J. Fixsen, and J. C. Mather, Astrophys.J. 581, 817-822 (2002).

14. S. Bashinsky, and U. Seljak, Phys.Rev. D69, 083002 (2004), astro-ph/ 0310198.

15. Z. Hou, R. Keisler, L. Knox, M. Millea, and C. Reichardt (2011), 1104.2333.

16. W. Hu, D. Scott, N. Sugiyama, and . White, Martin J., Phys.Rev. D52, 5498-5515 (1995), astro-ph/9505043.

17. E. Komatsu, et al., Astrophys.J.Suppl. 180, 330-376 (2009), 0803.0547.

18. M. Tortola, J. Valle, and D. Vanegas (2012), 1205 . 4018.

19. K. Ichikawa, M. Fukugita, and M. Kawasaki, Phys.Rev. D71, 043001 (2005), astro-ph/ 0409768.

20. D. Spergel, et al., Astrophys.J.Suppl. 170, 377 (2007), astro-ph/ 0603449.

21. A. D. Linde, Phys.Lett. B129, 177-181 (1983).

22. R. Easther, and L. McAllister, JCAP 0605, 018 (2006), hep-th/ 0512102.

23. L. Grishchuk, Sov.Phys.JETP 40, 409-415 (1975).

24. A. A. Starobinsky, JETP Lett. 30, 682-685 (1979). 
25. J. M. Maldacena, JHEP 0305, 013 (2003), astro-ph/ 0210603.

26. P. Creminelli, and M. Zaldarriaga, JCAP 0410, 006 (2004), astro-ph/ 0407059.

27. D. Seery, and J. E. Lidsey, JCAP 0506, 003 (2005), astro-ph/ 0503692.

28. C. Cheung, A. L. Fitzpatrick, J. Kaplan, and L. Senatore, JCAP 0802, 021 (2008), 0709.0295.

29. J. Ganc, and E. Komatsu, JCAP 1012, 009 (2010), 1006.5457.

30. S. Renaux-Petel, JCAP 1010, 020 (2010), 1008.0260.

31. P. Creminelli, G. D'Amico, M. Musso, and J. Norena, JCAP 1111, 038 (2011), 1106.1462.

32. I. Agullo, and L. Parker, Phys.Rev. D83, 063526 (2011), 1010.5766.

33. J. Ganc, Phys.Rev. D84, 063514 (2011), 1104.0244.

34. J. Ganc, and E. Komatsu (2012), 1204.4241.

35. E. Komatsu, and D. N. Spergel, Phys.Rev. D63, 063002 (2001), astro-ph/ 0005036. 